and so under the right circumstances large-scale lithospheric fractures may occur.

As interpreted by Oxburgh and Turcotte, the palaeomagnetic evidence suggests that, following a period of relative quiescence lasting most of the Mesozoic, the eastern part of Africa began to move northwards about 100 million years (Myr) ago at a rate of about $0.25^{\circ}$ per million years. The western part of Africa, by contrast, began to move much more slowly. Taking into account the drift rate and the size of the African plate as extrapolated backwards throughout the past $100 \mathrm{Myr}$, Oxburgh and Turcotte calculate that the tensional stress in the centre of a circular plate the size of the African plate (and drifting towards the equator) would be about 135 bar, which seems to be of the same order as the stresses associated with current continental faulting. In other words, it is quite conceivable that the East African rift system was produced by membrane stresses in the lithosphere, developed in response to the rapid latitude change of eastern Africa during the late Cretaceous and early Tertiary-and that is precisely what Oxburgh and Turcotte propose happened.

It follows from this interpretation that the East African rift system is probably a southward-propagating fracture system. There is little direct evidence on the age of the fracture itself; but it is implicit in a propagating fracture system that the onset of rift volcanism will migrate at the same rate as that at which the fracture extends. At most places along the East African rift, the volcanism, once started, has continued to the present; and so the onset of volcanism may be determined from the age of the oldest volcanic rocks available. Such age data from the eastern rift show that the locus of this onset has migrated southwards at a rate of about $0.23^{\circ}$ per million years. which is in excellent agreement with the palaeomagnetically-determined northward drift rate of the eastern part of the plate. Numbers apart, however, the advantage of regarding the East African rift as a propagating fracture rather than the locus of a hot spot is that so much more can be explained. The tensional features of the rift are an obvious case in point. Equally, hot spots seem incapable of explaining why volcanism continues at any given point once the plate has passed over the spot; a propagating fracture, on the other hand, would imply continued dilation and thus continued volcanism.

\title{
Internal magnetic field on Mercury?
}

from Peter J. Smith Geomagnetism Correspondent

Hitherto, it has not been considered likely that Mercury possesses an internal magnetic field. It is true that the planet has an unusually high density and thus by implication a large core; but the low rate of rotation would seem to preclude the generation of internal fields by dynamo action. Moreover, unlike Jupiter, Mercury has never produced any evidence of radio emissions which would indicate the presence of radiation belts.

The preliminary data from the Mariner 10 magnetic field survey near Mercury, now recorded by Ness et al. (Science, 185, 151; 1974), are thus surprising. For on March 29 , 1974 Mariner 10 passed within 704 $\mathrm{km}$ of Mercury's surface where it recorded a magnetic field of $98 \gamma$, which is about five times higher than the average interplanetary field in the vicinity. Moreover, both a well developed bow shock wave and a magnetosphere-like region were observed -- features comparable to those of the Earth, which has a global field, but completely alien to a body such as the Moon, which does not.

So do these results imply an in- ternal Mercurian field? They certainly imply it, but do not prove it because there are other processes, such as field induction by solar wind interaction, which could just explain the limited data yet available. Another Mercury encounter will be required to settle the matter; but in the meantime Ness and his colleagues interpret the whole evidence as generally favouring the intrinsic field idea. Approximate figures then suggest that the Mercurian dipole moment is about $4 \times 10^{-4}$ that of the Earth, that the dipole is close to the rotation axis and that it is offset by 0.47 Mercurian radii (not unacceptable in view of the large core).

A dynamo currently active within Mercury would indicate the viability of dynamo action under unexpected and unusual conditions. On the other hand, if the Mercurian field is due to remanent magnetism left from a dynamo now extinct, the implication must be that the planet once rotated much faster than it does now. In any event, internal planetary magnetic fields are rare enough to make the discovery of one within Mercury (if confirmed) a major advance in the exploration of the Solar System.
The Oxburgh-Turcotte model is, of course, an oversimplification. The rea! African plate is not circular, does not remain the same size, and does not undergo a simple change of curvature. Moreover, the unstressed radii of curvature are not constant over the plate but vary from element to element within it. The oceanic lithosphere within the plate is subject to thermal stresses as well as membrane stresses; the location of fracturing within continental lithosphere may be governed partly by previous history of faulting; even mid-plate areas will be affected to some extent by interactions with neighbouring plates; and so on. But these are points of detail, rather than principle, which do not deflect Oxburgh and Turcotte from their view that membrane stress effects are substantially more plausible than hot spot processes.

\section{Ribosome structure and function}

\section{from a Correspondent}

THE EMBO meeting on ribosome structure (Göteborg, Sweden, June 27-30) was a field day for Escherichia coli. The most popular topic was the three-dimensional arrangement of the ribosomal proteins, where several different approaches are being used with considerable success. C. Cantor (Columbia University, New York) has used a fluorescence method in which reconstituted $30 \mathrm{~S}$ ribosomes are labelled with two different fluorescent dyes on two specific proteins. By varying the proteins labelled, he has been able to classify many pairs of $30 \mathrm{~S}$ proteins as being close, or far apart in the ribosome.

Experiments with bifunctional protein cross-linking reagents were reported by several delegates, the most impressive being those of $\mathbf{R}$. Traut (University of California, Davis) who used the assymetric reagent 4-mercapto-butyrimidate to establish nearly twenty pairs of cross-linkable proteins from both subparticles. This reagent reacts first with $\varepsilon$ amino groups, leaving the thiol group free to react with protein sulphydryl groups under oxidising conditions.

G. Stöffler and G. Tischendorf (MaxPlanck-Institut, Berlin) have approached the topography question by electron microscopy. Since both subparticles have an assymetric shape, it is possible to localise the positions of proteins by examining the complexes formed with the ribosome by individual proteinspecific antibodies. So far twenty $50 \mathrm{~S}$ and seven $30 \mathrm{~S}$ proteins have been localised by this technique. H. Zeichhardt (Max-Planck-Institut, Berlin) used specific antibodies to examine the subparticle interface; although many 